\title{
Regulation of Chlamydia psittaci (Strain Guinea Pig Inclusion Conjunctivitis) Growth in McCoy Cells by Amino Acid Antagonism
}

\author{
By ANTHONY M. COLES* AND JOHN H. PEARCE \\ Department of Microbiology, University of Birmingham, Birmingham B15 2TT, UK
}

(Received 6 October 1986)

\begin{abstract}
Chlamydiae have amino acid requirements for growth in tissue culture as defined by those amino acids whose individual omission from the growth medium prevents chlamydial multiplication. We have tested the hypothesis that this inhibition of growth arises as a result of antagonism between particular amino acids such that inhibition occurs when the concentration of one amino acid is reduced in the presence of the antagonist amino acid at high concentration. Using the Chlamydia psittaci strain guinea pig inclusion conjunctivitis (GPIC), in the presence of cycloheximide, the requirement for valine was abrogated by the simultaneous omission of isoleucine, that for phenylalanine by simultaneous omission of tryptophan and that for leucine by simultaneous omission of isoleucine plus valine. The antagonism shown between leucine and isoleucine plus valine appears to be unique among bacteria. In the absence of cycloheximide, GPIC had an additional need for tryptophan, tyrosine and isoleucine; these amino acid requirements were shown for both infected McCoy, HeLa and BHK cells. The results are consistent with a mechanism for regulation of parasite growth which depends on the balance of amino acid concentrations in the extracellular environment.
\end{abstract}

\section{INTRODUCTION}

Chlamydiae are obligate parasitic bacteria that multiply within a membrane-bound vacuole in the cytoplasm of eukaryotic cells in vivo and in tissue culture. The cytoplasmic environment should provide an abundant source of nutrients, reducing the range of compounds synthesized by the parasite. High-energy intermediates, in particular ATP (Moulder, 1974; Hatch et al., 1982), are supplied by the host; the success of the parasite is therefore inexorably linked to the nutritional status of the host cell.

The exogenous amino acid supply can regulate chlamydial growth in tissue culture. Indeed, dormant (non-multiplying) infections can be established by infecting nutritionally deprived cells and the transition to productive infection can then be induced either by supplying exogenous amino acids (Bader \& Morgan, 1958) or by inhibiting host protein synthesis with cycloheximide (Hatch, 1975). More recent observations on amino acid requirements for growth of chlamydiae in cycloheximide treated cell cultures have shown that, for Chlamydia trachomatis strains, patterns of requirement correlated with clinical syndrome and, for $C$. psittaci strains, with animal host origin. This suggests that chlamydiae may have adapted to different nutritional environments (Allan \& Pearce, 1983b).

In these studies detailed examination of the amino acid requirements of the guinea pig inclusion conjunctivitis strain of $C$. psittaci (GPIC) revealed that threshold concentrations in culture medium of 80,80 and approximately $8 \mu \mathrm{M}$ for leucine, valine and phenylalanine, respectively, were needed for chlamydial multiplication compared with the values of 400,400 and $200 \mu \mathrm{M}$ found in the Eagle's minimal essential medium (MEM) used for routine culture

\footnotetext{
Abbreviations: FBS, foetal bovine serum; GPIC, guinea pig inclusion conjunctivitis strain of Chlamydia psittaci; i.f.u., inclusion-forming units; MEM, minimal essential medium; p.i., post-infection.
} 
(Allan \& Pearce, 1983a). Analysis of these amino acids in supernatant medium from infected McCoy cells that had been cycloheximide treated and irradiated showed that there was no detectable consumption during multiplication, indicating that the content of host intracellular amino acid pools was sufficient for parasite growth. To account for the phenomenon of growth inhibition on deprivation of extracellular amino acid(s) it was proposed that a regulatory or physiological mechanism operated which restricted the availability of intracellular amino acid(s) and which depended on relative concentrations of amino acids interacting in competiton (Allan \& Pearce, 1983a).

Competition or antagonism between amino acids may affect growth by the allosteric regulation of biosynthetic pathways or, as envisaged for this system, by the modulation of amino acid transport across either the parasite envelope or the host-derived vesicle membrane surrounding the parasite. For example, if valine is removed from the medium, a residual concentration will remain in the host cytoplasm. If valine and isoleucine share a common transport pathway into the parasite, residual valine in the host cytoplasm may not be transported due to the presence of a relatively high concentration of isoleucine in the culture medium and a correspondingly high concentration in the host cytoplasm. Thus, isoleucine would antagonize transport of valine into the parasite. It would then be expected that reduction of the isoleucine concentration in the culture medium would abrogate the inhibition of valine transport and so restore chlamydial multiplication.

It follows from the above hypothesis that inhibition of parasite growth can arise after amino acid deprivation not simply because the parasite cannot synthesize the amino acid but because the effect of deprivation, which may leave intracellular amino acid sufficient for parasite needs when cycloheximide is present, is greatly increased by the presence of the antagonist amino acid at higher concentration. Imbalance of certain amino acids should then serve to regulate parasite growth in the host cell with relief from inhibition being achieved either by restoration of the deficient amino acid or by additional deprivation of its antagonist. The present paper describes the use of this latter approach to seek evidence of antagonism between amino acids and, if obtained, to determine which amino acids or combinations of amino acids were mutually antagonistic.

\section{METHODS}

Organism. Chlamydia psittaci (strain GPIC) was grown in irradiated McCoy cells as described previously (Allan $\&$ Pearce, 1982) except that monolayers were established in six-well dishes (Flow Laboratories) and infected by centrifugation $\left(1200 \mathrm{~g}, 60 \mathrm{~min}, 36^{\circ} \mathrm{C}\right)$ using micro-titre plate carriers. Organisms were purified using Urografin 370 (Schering) and stored at $-70^{\circ} \mathrm{C}$ in PBS ( $0.17 \mathrm{M}-\mathrm{NaCl}, 3.3 \mathrm{mM}-\mathrm{KCl}, 10 \mathrm{~mm}-\mathrm{Na}_{2} \mathrm{HPO}_{4}, 1.8 \mathrm{~mm}-\mathrm{KH}_{2} \mathrm{PO}_{4}$; pH 7.4) containing 0.25 M-sucrose (Howard et al., 1974).

Cell culture. McCoy cells were cultured according to the method of Griffiths et al. (1976) using Eagle's MEM (Gibco) containing foetal bovine serum (FBS; $5 \%, \mathrm{v} / \mathrm{v})$, glutamine ( $2 \mathrm{mM}$ ) and streptomycin $\left(0 \cdot 1 \mathrm{mg} \mathrm{ml}^{-1}\right)$. HeLa 229 or BHK cells were cultured in MEM containing FBS $(10 \%, \mathrm{v} / \mathrm{v})$, glutamine $(2 \mathrm{mM})$ and streptomycin $\left(0.1 \mathrm{mg} \mathrm{ml}^{-1}\right)$. Cells were judged to be free of mycoplasma contamination by staining with the DNA fluorochrome Hoechst 33258 and examination by ultraviolet microscopy.

Infectivity titration. Infectivity of GPIC was titrated using McCoy, HeLa 229 or BHK cells as described by Griffiths et al. (1976) for McCoy cells except that all cultures were unirradiated unless stated otherwise. At $26 \mathrm{~h}$ post-infection (p.i.) monolayers were fixed, stained with Giemsa and examined microscopically for inclusion formation. Infection was assessed as inclusion-forming units (i.f.u.) over 30 microscope fields (approximately 2500 cells) in duplicate monolayers with about $10 \%$ cells infected when maintained in complete medium. Infectivity values quoted in the text are means of two or more experiments.

Infectivity of GPIC under conditions of amino acid deprivation. In certain experiments the concentrations of single amino acids or combinations of amino acids in the growth medium were altered. On these occasions growth media were prepared from a 'Select Amine' kit (Gibco) using the Earle's balanced salts solution, vitamins and 13 amino acid solutions provided in the kit to reconstitute Eagle's MEM (Allan \& Pearce, 1983a). Amino acid concentrations were as in Eagle's MEM except that in test media, single amino acids or combinations of amino acids were either omitted or present at reduced concentrations. All media were then supplemented with streptomycin $\left(0.1 \mathrm{mg} \mathrm{ml}^{-1}\right)$ and dialysed FBS [ $5 \%(\mathrm{v} / \mathrm{v}) ; 300 \mathrm{ml}$ serum dialysed against $3 \times 51$ PBS and sterilized by membrane filtration (Millipore, $0.22 \mu \mathrm{m}$ pore size)]; in certain experiments cycloheximide $\left(1 \mu \mathrm{g} \mathrm{ml}^{-1}\right)$ was also present in media. 
Values for infectivity of GPIC under conditions of amino acid deprivation were calculated relative to those obtained using a fully supplemented control medium prepared as described for the test media but containing all 13 amino acids. The control medium appeared identical to the commercially supplied Eagle's MEM after supplementation, as judged by the infectivity titre.

\section{RESULTS}

\section{Effects of irradiation, cycloheximide and host cell type on GPIC infectivity after amino acid deprivation}

In previous work Allan \& Pearce (1983a) defined amino acid requirements using irradiated McCoy cells treated with cycloheximide. Irradiation and cycloheximide treatment interfere with host protein synthesis, reducing amino acid utilization and increasing the availability of amino acids to the parasite; amino acid pool sizes may also vary according to the host cell type. The influence of these variables on the inhibition of parasite growth after the omission of single amino acids was therefore assessed.

Growth of GPIC in McCoy cells in the presence of cycloheximide required the presence of leucine, phenylalanine and valine whether irradiated or non-irradiated host cells were used (Table 1); similar results were obtained for growth in non-irradiated HeLa or BHK cells. On omission of cycloheximide from irradiated McCoy and non-irradiated McCoy, HeLa and BHK cells, inclusion formation required the additional presence of isoleucine, tryptophan and tyrosine (Table 1). Where omission largely inhibited growth, very small inclusions were occasionally detected in host cells, as observed in earlier work (Allan \& Pearce, 1983a, $b$ ). In a number of instances omission of certain amino acids actually stimulated growth of GPIC. This was most marked with McCoy and BHK cells but was not consistently maintained for any given amino acid with all three cell types. Non-irradiated McCoy cells treated with cycloheximide were used in all further experiments described in the following sections.

\section{Effect of valine deprivation on the threshold concentration of leucine needed in the growth medium for chlamydial multiplication}

The hypothesis that apparent amino acid requirements arose from competition between specific amino acids was examined by looking for a decrease in the threshold concentration (that needed for chlamydial infectivity approximating to the infectivity observed in fully supplemented medium) of a required amino acid when a putative competitor amino acid was either present at decreased concentration or omitted altogether. Valine and leucine are structurally similar and have a common transport system in Escherichia coli (Piperno \& Oxender, 1968). The effect of decrements in the valine concentration on the leucine threshold for GPIC growth in McCoy cells was therefore investigated. The concentrations of leucine, valine and phenylalanine in MEM were 400,400 and $200 \mu \mathrm{M}$, respectively (Table 1). With $200 \mu \mathrm{M}$-valine, the leucine threshold was observed to be $80 \mu \mathrm{M}$ and remained at this value when the valine concentration was reduced first to $100 \mu \mathrm{M}$ and then to $50 \mu \mathrm{M}$. Very small inclusions were occasionally visible at $40 \mu \mathrm{M}$-leucine in the presence of valine at either 200,100 or $50 \mu \mathrm{M}$. Similar experiments failed to demonstrate a decrease in the valine threshold with decrements in leucine concentration. Mutual antagonism could thus not be detected between leucine and valine.

\section{Effect of group deletion of amino acids on the valine threshold}

The possibility that other amino acids antagonized valine was examined by determination of the valine threshold for GPIC growth in McCoy cells in the absence of groups of amino acids which were not essential for GPIC growth in the presence of cycloheximide (Table 1). As in the previous experiments with leucine, a decreased valine threshold was expected on removal of an amino acid which was antagonistic to valine. The threshold concentration of valine for growth of GPIC when all other amino acids in MEM were present at their normal concentrations was found to be $60 \mu \mathrm{M}$, approximating to the value of $80 \mu \mathrm{M}$ previously observed (Allan \& Pearce, $1983 a$ ). Omission of the amino acid group arginine, tryptophan and tyrosine had no effect on the valine threshold although it slightly reduced the proportion of cells infected (Table 2). When the 
Table 1. Effects of irradiation, cycloheximide and host cell type on the growth of GPIC after omission of single amino acids from the growth medium

Infection was assessed at $26 \mathrm{~h}$ p.i. in McCoy, HeLa or BHK cell monolayers, either irradiated or nonirradiated, and either in the presence or in the absence of cycloheximide. Amino acid concentrations $(\mu \mathrm{M})$ in the complete medium are shown in parentheses. Infectivity values are means of two to four experiments. Infection is expressed as a percentage of the number of infected cells in the control receiving complete medium. $\mathrm{CH}$, cycloheximide; I, irradiated; NI, non-irradiated.

\begin{tabular}{|c|c|c|c|c|c|c|c|c|}
\hline \multirow{3}{*}{$\begin{array}{l}\text { Amino acid } \\
\text { omitted }\end{array}$} & \multicolumn{8}{|c|}{ Infection in treated cell cultures $(\%)$} \\
\hline & \multicolumn{2}{|c|}{ McCoy (I) } & \multicolumn{2}{|c|}{$\operatorname{McCoy}(\mathrm{NI})$} & \multicolumn{2}{|c|}{ HeLa (NI) } & \multicolumn{2}{|c|}{ BHK (NI) } \\
\hline & $+\mathrm{CH}$ & $-\mathrm{CH}$ & $+\mathrm{CH}$ & $-\mathrm{CH}$ & $+\mathrm{CH}$ & $+\mathrm{CH}$ & $+\mathrm{CH}$ & $-\mathrm{CH}$ \\
\hline None & 100 & 100 & 100 & 100 & 100 & 100 & 100 & 100 \\
\hline Arginine (656) & 83 & 202 & 85 & 357 & 88 & 153 & 139 & 70 \\
\hline Cystine (260) & 82 & 33 & 88 & 109 & 82 & 94 & 338 & 91 \\
\hline Glutamine $(2000)$ & 44 & 248 & 67 & ND & 56 & ND & ND & ND \\
\hline Histidine (200) & 81 & 136 & 88 & 126 & 99 & 79 & 94 & 68 \\
\hline Isoleucine $(400)$ & 91 & $0^{*}$ & 106 & 0 & 56 & 0 & 537 & 0 \\
\hline Leucine (400) & 0 & 0 & 0 & 0 & 0 & 0 & 0 & 0 \\
\hline Lysine (400) & 80 & 157 & 77 & ND & 99 & ND & ND & ND \\
\hline Methionine (128) & 79 & $188 \dagger$ & 91 & 334 & 97 & 62 & 70 & 605 \\
\hline Phenylalanine (193) & 0 & 0 & 0 & 0 & $0^{*}$ & 0 & 0 & 0 \\
\hline Threonine (403) & 85 & $127 \dagger$ & 79 & 213 & 73 & 107 & 386 & 483 \\
\hline Tryptophan (49) & 94 & $0^{*}$ & 99 & 0 & 61 & 0 & 81 & 0 \\
\hline Tyrosine (199) & 95 & 0 & 92 & 0 & 70 & 0 & 211 & 0 \\
\hline Valine (393) & 0 & 0 & 0 & 0 & 0 & 0 & 0 & 0 \\
\hline
\end{tabular}

ND, Not done.

* Very small inclusions (groups of approx. five particles) were occasionally visible in host cell cytoplasm. $\dagger$ Small inclusions.

Table 2. Effect of deletion of groups of amino acids on the threshold concentration of valine needed for unimpaired infection by GPIC

Infection was assessed at $26 \mathrm{~h}$ p.i. and is expressed as a percentage of the number of infected cells in the control receiving complete medium. Values are means of two experiments.

$\begin{array}{lcc}\begin{array}{c}\text { Amino acids } \\ \text { omitted }\end{array} & \begin{array}{c}\text { Valine concn } \\ (\mu \mathrm{M})\end{array} & \begin{array}{c}\text { Infection } \\ (\%)\end{array} \\ \text { None } & 400 & 100 \\ \text { Arginine, tryptophan } & 60 & 78 \\ \text { and tyrosine } & 20 & 0^{*} \\ & 10 & 0 \\ \text { Lysine, methionine } & 60 & 83 \\ \text { and threonine } & 20 & 32 \\ & 10 & 0^{*} \\ \text { Cystine, glutamine, } & 60 & 31 \\ \text { histidine and } & 20 & 30 \\ \text { isoleucine } & 10 & 42\end{array}$

* Very small inclusions were occasionally visible in host cells.

group lysine, methionine and threonine was omitted a proportion of cells showed inclusions at $20 \mu \mathrm{M}$-valine and occasional very small inclusions were visible at $10 \mu \mathrm{M}$-valine (Table 2), suggesting that one or a combination of these amino acids weakly antagonized valine. In contrast, omission of cystine, glutamine, histidine and isoleucine as a group decreased the valine threshold to below $10 \mu \mathrm{M}$ (the lowest valine concentration tested). The low value of $31 \%$ infection at $60 \mu \mathrm{M}$-valine was probably due to the omission of glutamine, whose deletion 
Table 3. Effect of omission of either cystine, glutamine, histidine or isoleucine on the threshold concentration of valine needed for unimpaired infection by GPIC

Infection was assessed at $26 \mathrm{~h}$ p.i. and is expressed as a percentage of the number of infected cells in the control receiving complete medium. Values are means from one experiment typical of three experiments.

$\begin{array}{lcc}\begin{array}{c}\text { Amino acid } \\ \text { omitted }\end{array} & \begin{array}{c}\text { Valine concn } \\ (\mu \mathrm{M})\end{array} & \begin{array}{c}\text { Infection } \\ (\%)\end{array} \\ \text { None } & 400 & 100 \\ \text { Cystine } & 60 & 121 \\ & 20 & 28^{\dagger} \\ \text { Glutamine } & 10 & 0 \\ & 60 & 59 \\ \text { Histidine } & 20 & 0^{*} \\ & 10 & 0^{*} \\ \text { Isoleucine } & 60 & 80 \\ & 20 & 0 \\ & 10 & 0 \\ & 60 & 110 \\ & 20 & 121 \\ & 10 & 121\end{array}$

* Very small inclusions were occasionally visible in host cells.

$\uparrow$ Small inclusions.

consistently yielded reduced numbers of inclusions in previous experiments (Table 1) and which is known to fall significantly in extracellular concentration during incubation with uninfected or infected cells (Allan \& Pearce, 1983a).

The decrease in threshold concentration of valine observed indicated that the antagonist of valine was one or a combination of the amino acids in the group cystine, glutamine, histidine and isoleucine. This was resolved by analysing the effect of absence of single members of the group. Omission of cystine alone yielded small inclusions at $20 \mu \mathrm{M}$-valine, but none at $10 \mu \mathrm{M}$ valine (Table 3). Deprivation of cystine thus reduced the valine threshold but not to the level observed when the entire group of four amino acids was omitted $(<10 \mu \mathrm{M})$ (Table 2). Omission of glutamine or histidine did not alter the valine threshold of $60 \mu \mathrm{M}$, although in the case of glutamine the number of cells infected at this concentration was reduced to $59 \%$ of that in the fully supplemented medium, as in previous experiments. However, the omission of isoleucine slightly increased inclusion formation and reduced the valine threshold to $10 \mu \mathrm{M}$ (the lowest valine concentration tested) (Table 3). This experiment identified isoleucine as the antagonist of valine.

\section{Detection of amino acid antagonism by co-omission of agonist and antagonist amino acids}

Examination of amino acid thresholds provided a sensitive method of identifying antagonists, but was laborious for tests of many combinations of amino acids. Omission of pairs of amino acids was therefore investigated as a test for antagonism. The omission of valine plus isoleucine, as agonist and antagonist respectively, should permit inclusion formation given the evidence that inhibition of growth of GPIC resulting from reduction in valine concentration was abrogated by the further omission of isoleucine (Table 3). Using this modified test only isoleucine of the 12 remaining amino acids present in MEM, when omitted with valine, led to the restoration of GPIC infectivity ( $87 \%$ of the fully supplemented medium; two experiments). By the same procedure, only the omission of tryptophan with phenylalanine led to GPIC infectivity $(93 \%$ of the fully supplemented medium; two experiments), identifying tryptophan as the antagonist of phenylalanine. However, in the case of leucine, co-omission with any other single amino acid failed to restore infectivity, suggesting either that antagonism of leucine did not occur or that more than one amino acid acted as antagonist. 


\section{Detection of the leucine antagonist}

Further experiments on the omission of groups of amino acids (those specified in Table 2) failed to identify the leucine antagonist. The possibility was therefore considered that multiple combinations of amino acids other than those tested might be required for the antagonism of leucine. Accordingly leucine, valine, isoleucine, phenylalanine and tryptophan were omitted as a group. Inclusions were produced at a level identical to that of the fully supplemented control, indicating that a combination of two or more of the amino acids valine, isoleucine, phenylalanine and tryptophan antagonized leucine. Leucine plus two of valine, isoleucine, phenylalanine and tryptophan were then omitted. Only omission of the group leucine, valine and isoleucine permitted growth of GPIC ( $98 \%$ of that of the fully supplemented medium; two experiments). In further experiments leucine plus additional combinations of amino acids present in MEM were omitted together but none permitted growth of GPIC. Thus valine plus isoleucine were identified as the sole co-antagonists of leucine.

\section{DISCUSSION}

The data presented here are consistent with a mechanism of growth regulation which depends on antagonism between specific amino acids so that the balance of amino acid levels, i.e. their relative rather than their absolute concentrations, controls chlamydial multiplication. For $C$. psittaci strain GPIC, antagonism operates between phenylalanine and tryptophan, between valine and isoleucine, and between leucine and valine plus isoleucine. The appearance, in certain experiments, of small inclusions without reduction in inclusion number may reflect minor limitation of chlamydial growth by the omitted amino acid. The presence of occasional very small inclusions where amino acid omission almost completely inhibited growth appears likely to have been due to incomplete antagonism, probably because of variations in intracellular pool sizes from cell to cell.

Whilst amino acid deprivation leading to growth inhibition may be said to define 'apparent' requirements, it is evident that failure of the parasite to synthesize amino acids is also involved. Adequate parasite synthesis would restore amino acid levels within the chlamydial cytoplasm when a deficiency is created as a result of antagonism.

The lack of effect of host cell irradiation and the similarity of McCoy, HeLa 229 and BHK cells in their susceptibility to GPIC during amino acid deprivation supports the conclusion from previous work on different strains (Allan \& Pearce, 1983b) that the amino acids required for chlamydial growth are specified by the parasite rather than the host. However, as emphasized previously (Allan \& Pearce, 1983 b), the data obtained here or in earlier analyses do not define the complete requirements of a chlamydial strain. This is underlined by the evidence that withdrawal of cycloheximide added isoleucine, tryptophan and tyrosine to the list of required amino acids for growth of GPIC. By the same reasoning advanced earlier, these latter requirements may arise from the reduction in amino acid pool sizes as a result of increased host utilization in the absence of cycloheximide. Thus, deprivation of, say, tryptophan, would now lead to inhibition of growth, because the intracellular level of tryptophan has been lowered, via host protein synthesis, to a point where tryptophan can be successfully antagonized by intracellular phenylalanine whose extracellular concentration remains high. If, as postulated earlier, the antagonism occurs at a transport system then the transport system must be relatively specific for these two amino acids. Phenylalanine and tryptophan are present in MEM at 200 and $50 \mu \mathrm{M}$, respectively (Table 1). If these approximate to intracellular ratios in the presence of cycloheximide then the transport system must have a significantly greater affinity for tryptophan than phenylalanine, since omission of phenylalanine leads to inhibition of growth (via antagonism by tryptophan) whereas omission of tryptophan does not. Similar arguments apply to valine versus isoleucine and leucine versus isoleucine plus valine.

It would appear that for GPIC, cycloheximide withdrawal defines the antagonists for those amino acids identified as 'required' by the omission of single amino acids in the presence of cycloheximide. The specificity of these interactions, if mediated at the level of transport, strongly affirms their bacterial origin given the known low specificity of mammalian transport 
systems (Oxender et al., 1977). Leucine, isoleucine and valine share a transport system in E. coli, as do phenylalanine, tryptophan and tyrosine (Anraku, 1980). Competition between leucine versus valine plus isoleucine may be unique to Chlamydia. It is possible that tyrosine, which was not shown to antagonize leucine, valine or phenylalanine, may have a minor undetected role in phenylalanine antagonism.

Whilst the evidence suggests that these phenomena are parasite- rather than host-dependent, this does not conclusively rule out their mediation at either the host-derived vesicle membrane or the host plasma membrane since either could be subject to modification by the parasite, either by glycosylation (Stokes, 1974), or possibly by the insertion of chlamydial surface antigen (Richmond \& Stirling, 1981). However, two lines of evidence indicate that the host plasma membrane is unlikely to be the site of antagonism. First, Allan \& Pearce (1983a) showed in preliminary experiments that the association of leucine with the host cell was not inhibited below its threshold concentration in the presence of the 12 other amino acids. Second, the inhibition generated by the omission of one extracellular amino acid could be relieved simply by the withdrawal of the competing amino acid from the medium. It follows that the initial antagonism must have occurred, not at the plasma membrane, but at an intracellular site. Indeed, experiments in progress to define the transport characteristics of isolated reticulate body (reproductive) forms of chlamydiae suggest that the site of antagonism is at the chlamydial envelope.

There are two important ways in which amino acid antagonism may have been modified in the experiments described here. First, host transport mechanisms may have altered the intracellular levels of amino acids after the omission of extracellular amino acid(s). How far this might influence intracellular levels of, say, valine, on deprivation of extracellular valine and isoleucine needs to be determined in further experiments. Chlamydial growth is clearly affected by host properties mediating amino acid levels, as indicated by the differing stimulation of growth in McCoy, HeLa and BHK cells on amino acid omission (Table 1). Second, it is possible that chlamydiae can, in fact, synthesize some required amino acids and that their biosynthesis is itself subject to antagonism. This is well established for $E$. coli $\mathrm{K} 12$ where, for example, growth can be inhibited by the prevention of isoleucine synthesis, via the synthetic pathway for branched chain amino acids, after the addition of valine in high concentration; growth can then be restored by the further addition of isoleucine (De Felice et al., 1979). Only biosynthesis of arginine and lysine has so far been demonstrated for C. psittaci (Treuhaft \& Moulder, 1968; Moulder et al., 1963) and the establishment of the presence or absence of pathways for the synthesis of branched chain or aromatic amino acids must be an important objective of future research.

The observations presented here establish the importance of relative amino acid concentrations in determining chlamydial growth in cell culture. Of those amino acids so far examined, valine and leucine show the highest threshold concentrations $(60-80 \mu \mathrm{M}$, relative to $400 \mu \mathrm{M}$ for valine, leucine and isoleucine in medium). Thus a fall of five- to sevenfold in the concentration of valine or leucine relative to isoleucine is sufficient to inhibit growth completely. It follows that selective increase in the extracellular concentration of isoleucine should also inhibit chlamydial growth and this has been observed in recent studies (unpublished observations).

The concept of the regulation of parasite growth by amino acid balance is, to our knowledge, novel, and is potentially applicable to any intracellular microbe that possesses transport mechanisms but lacks the ability to synthesize amino acids. Extreme imbalance in amino acid levels in vivo appears unlikely to occur in the normal host. Alterations sufficient to slow parasite growth rate and restrict the numbers of progeny in infected cells would seem more feasible. However, in the infected host, the induction of interferon is associated with major activation of the tryptophan-degrading enzyme indoleamine dioxygenase (Yoshida et al., 1979, 1981; Hayaishi, 1985). Studies in tissue culture indicate that interferon activation may lead to the inhibition of growth of both Toxoplasma gondii (Pfefferkorn, 1984) and C. psittaci (Byrne et al., 1986) by the depletion of tryptophan. In such situations aromatic amino acid imbalance may be the dominant factor in the inhibition of parasite growth. 
We are indebted to the Medical Research Council for financial support.

\section{REFERENCES}

Allan, I. \& Pearce, J. H. (1982). Radiolabelling of Chlamydia psittaci (strain guinea pig inclusion conjunctivitis) to high specific activity using ${ }^{14} \mathrm{C}$ labelled amino acids. FEMS Microbiology Letters 13, 69-73.

Allan, I. \& Pearce, J. H. (1983a). Differential amino acid utilization by Chlamydia psittaci (strain guinea pig inclusion conjunctivitis) and its regulatory effect on chlamydial growth. Journal of General Microbiology 129, 1991-2000.

Allan, I. \& Pearce, J. H. (1983b). Amino acid requirements of strains of Chlamydia trachomatis and C. psittaci growing in McCoy cells: relationship with clinical syndrome and host origin. Journal of General Microbiology 129, 2001-2007.

ANRAKU, Y. (1980). Transport and utilization of amino acids by bacteria. In Microorganisms and Nitrogen Sources, pp. 9-33. Edited by J. W. Payne. Chichester: Wiley.

Bader, J. P. \& Morgan, M. D. (1958). Latent viral infection of cells in tissue culture. VI. Role of amino acids, glutamine and glucose in psittacosis virus propagation in L cells. Journal of Experimental Medicine, 108, 617-629.

BYRNe, G. I., LeHMANN, L. K. \& LANDRY, G. L. (1986). Induction of tryptophan catabolism is the mechanism for gamma-interferon-mediated inhibition of intracellular Chlamydia psittaci replication in T24 cells. Infection and Immunity 53, 347-351.

De Felice, M., Levinthal, M., Iaccarino, M. \& Guardiola, J. (1979). Growth inhibition as a consequence of antagonism between related amino acids: effect of valine in Escherichia coli $\mathrm{K}-12$. Microbiological Reviews 43, 42-58.

Griffiths, M. S., Ainsworth, S. \& Pearce, J. H. (1976). Infectivity titration of guinea-pig inclusion conjunctivitis agent in irradiated McCoy cells. Journal of General Microbiology 95, 249-256.

HATCH, T. P. (1975). Competition between Chlamydia psittaci and cells for host isoleucine pools: a limiting factor in chlamydial multiplication. Infection and Immunity 12, 211-220.

Hatch, T. P., Al-Hossainy, E. \& Silverman, J. L. (1982). Adenine nucleotide and lysine transport in Chlamydia psittaci. Journal of Bacteriology 150, 662670 .
HAYAISHI, O. (1985). Indoleamine 2,3-dioxygenase with special reference to the mechanism of interferon action. Biken Journal 28, 39-49.

Howard, L., Orenstein, N. S. \& KING, N. W. (1974). Purification on Renografin density gradients of Chlamydia trachomatis grown in the yolk-sac of eggs. Applied Microbiology 95, 249-256.

MOULDER, J. W. (1974). Intracellular parasitism: life in an extreme environment. Journal of Infectious Diseases 130, 300-306.

Moulder, J. W., Novosel, D. L. \& TribBy, I. C. (1963). Diaminopimelic acid decarboxylase of the agent of meningopneumonitis. Journal of Bacteriology 85, 701-706.

OXender, D. L., Lee, M., Moore, P. A. \& Cecchini, G. (1977). Neutral amino acid transport systems of tissue culture cells. Journal of Biological Chemistry 252, 2675-2679.

PFEFFERKORN, E. R. (1984). Interferon- $\gamma$ blocks the growth of Toxoplasma gondii in human fibroblasts by inducing the host cells to degrade tryptophan. Proceedings of the National Academy of Sciences of the United States of America 81, 908-912.

Piperno, J. R. \& OXender, D. L. (1968). Amino acid transport systems in Escherichia coli K12. Journal of Biological Chemistry 243, 5914-5920.

RICHMOND, S. J. \& STIRLING, P. (1981). Localization of chlamydial group antigen in McCoy cell monolayers infected with Chlamydia trachomatis or Chlamydia psittaci. Infection and Immunity 34, 561-570.

STOKES, G. (1974). Cycloheximide-resistant glycosylation in $\mathrm{L}$ cells infected with Chlamydia psittaci. Infection and Immunity 9, 497-499.

Treuhaft, M. W. \& Moulder, J. W. (1968). Biosynthesis of arginine in L-cells infected with chlamydiae. Journal of Bacteriology 96, 2004-2011.

Yoshida, R., URade, Y., ToKuda, M. \& HaYaishi, O. (1979). Induction of indoleamine 2,3-dioxygenase in mouse lung during virus infection. Proceedings of the National Academy of Sciences of the United States of America 76, 4084-4086.

Yoshida, R., Imanishi, J., OKU, T., Kishida, T. \& HAYAISHI, O. (1981). Induction of pulmonary indoleamine 2,3-dioxygenase by interferon. Proceedings of the National Academy of Sciences of the United States of America 78, 129-132. 